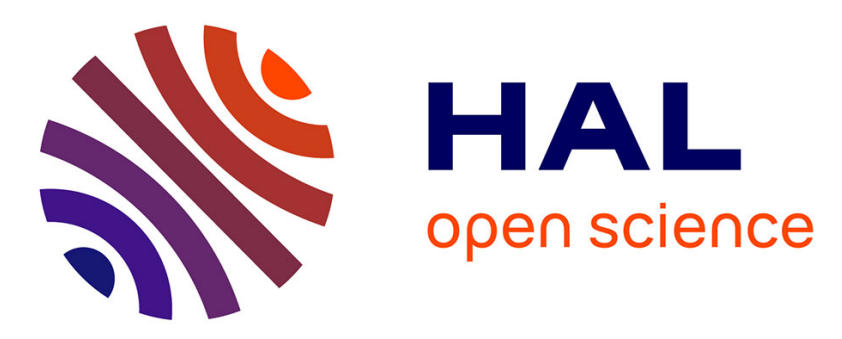

\title{
Investigation of arc dynamics during vacuum arc remelting of a Ti64 alloy using a photodiode based instrumentation
}

Pierre-Olivier Delzant, P Chapelle, Alain Jardy, Julien Jourdan, Yvon Millet

\section{- To cite this version:}

Pierre-Olivier Delzant, P Chapelle, Alain Jardy, Julien Jourdan, Yvon Millet. Investigation of arc dynamics during vacuum arc remelting of a Ti64 alloy using a photodiode based instrumentation. Journal of Materials Processing Technology, 2019, 266, pp.10-18. 10.1016/j.jmatprotec.2018.10.031 . hal-02106969

\section{HAL Id: hal-02106969 \\ https://hal.science/hal-02106969}

Submitted on 13 Jan 2022

HAL is a multi-disciplinary open access archive for the deposit and dissemination of scientific research documents, whether they are published or not. The documents may come from teaching and research institutions in France or abroad, or from public or private research centers.
L'archive ouverte pluridisciplinaire HAL, est destinée au dépôt et à la diffusion de documents scientifiques de niveau recherche, publiés ou non, émanant des établissements d'enseignement et de recherche français ou étrangers, des laboratoires publics ou privés. 


\title{
Investigation of arc dynamics during vacuum arc remelting of a Ti64 alloy using a photodiode based instrumentation
}

\author{
Pierre-Olivier Delzant ${ }^{1,2}$, Pierre Chapelle ${ }^{1}$, Alain Jardy ${ }^{1}$, Julien Jourdan ${ }^{1}$, Julien Jourdan ${ }^{2}$, Yvon Millet ${ }^{2}$ \\ ${ }^{1}$ Institut Jean Lamour, UMR 7198 CNRS/Université de Lorraine, LabEx DAMAS, \\ BP 50840, 54011 Nancy Cedex, France \\ ${ }^{2}$ TIMET Savoie, Avenue Paul Girod, 73400 Ugine, France
}

Corresponding author: P. Chapelle, Tel: +33 372742719, E-mail address: pierre.chapelle@univ-lorraine.fr

\begin{abstract}
Understanding the arc dynamics during VAR is important for controlling the final quality of the ingot, as the spatial and temporal distribution of the arc dictates the electric current and energy inputs at the ingot top, which in turn affect the hydrodynamics of the metal in the molten pool and the solidification conditions of the ingot. The arc dynamics during vacuum arc remelting was quantitatively investigated based on the analysis of the light emitted by the arc and reflected by the molten pool using photodiodes placed inside a full-scale furnace. The measurements allowed us to determine the location of the arc luminosity centroid and to follow its displacement as a function of time. They were made during the melt of a Ti64 alloy with an imposed alternated electromagnetic stirring of high intensity. The arc dynamics was found to be most of the time periodic, asymmetrical, and strongly correlated to the evolution of the stirring magnetic field. The arc centroid exhibited three regular displacement patterns throughout the melt. A common characteristic to all three patterns was the relative stability of the centroid during stirring plateaus and its rapid motion during stirring ramps. An evolution towards a much more centered distribution of the centroid was observed with the arc power decrease during the hot-topping phase. Possible implications of the observed arc dynamics on the final quality of the VAR ingot are discussed.
\end{abstract}

Keywords: Vacuum Arc Remelting, Titanium Alloy, Electric Arc, Dynamics, Photodiodes

\section{Introduction}

Vacuum Arc Remelting (VAR) is a metallurgical process used for the production of high quality ingots of special steels, nickel-based alloys, as well as titanium and zirconium alloys (Choudhury 1990). Among its 
benefits, this process allows for a good control of the solidification structure and the chemical homogeneity of the ingot. The VAR process, as illustrated in figure 1, consists of remelting under a high vacuum, a consumable metallic electrode into a secondary ingot that forms into a water-cooled copper crucible. The energy necessary for the melt is provided by an electric arc maintained between the electrode tip (operating as the cathode) and the top surface of the ingot (operating as the anode).

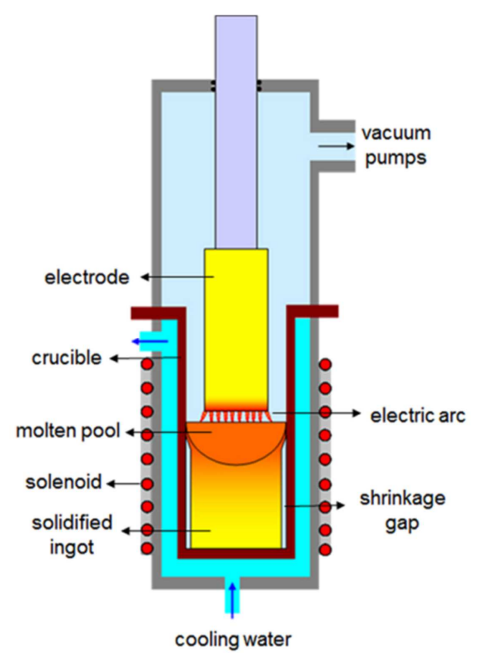

Figure 1: VAR process schematic representation.

In order to stabilize the arc, it can be confined with the aid of a magnetic field created by an external induction coil. The arc confinement is verified by security cameras (usually two diametrically opposed cameras) which face the ingot liquid pool through the gap formed between the electrode and the crucible. This magnetic field is also used to stir the liquid metal. By reversing periodically the coil current, stirring can be alternated. The dynamics of the electric arc indirectly impacts, through the distributions of energy and current density produced by the arc at the surface of the molten pool, the conditions of solidification of the ingot, hence the quality of the final product (Shevchenko and Ward 2008). Indeed, the distributions of energy and current density control the hydrodynamics of the liquid metal in the pool (which is governed by buoyancy forces and electromagnetic forces caused by the interaction of the flow of current through the pool with the self-induced and imposed magnetic fields), and in this way influence the dimensions and shape of the pool, the distributions of temperature and solute concentrations, and the stability of the solidification front. By controlling the thermal conditions 
at the ingot top, the electric arc influences also the size of the solidified metal region formed at the periphery of the ingot in contact with the crucible wall, which may affect the ingot sidewall quality.

The electric arc has been studied experimentally at two different time scales. At small time scales (less than a few milliseconds), direct visualization experiments performed in specifically instrumented furnaces, during the melt of Inconel 718 electrodes (Zanner 1979a), Zr electrodes (Chapelle et al. 2000), and Ti electrodes (Chapelle et al. 2014), have shown that the arc operates in a diffuse regime. The arc is composed of a metal vapor plasma that is provided by numerous cathode spots moving over the whole surface of the cathode. In the case of Ti alloys, the size of a spot was estimated to be several hundred micrometers and its apparent velocity over the cathode surface turned out to be of the order of 1 to 10 $\mathrm{m} / \mathrm{s}$.

Experiments dealing with large time scales (typically a few tens of seconds) have mostly focused on the overall dynamics of the electric arc resulting from the continuous creation, motion and extinguishment of cathode spots. Two kinds of experimental approach have been used. The first approach consists of analyzing the distribution of the magnetic field, induced by the arc current, outside the furnace. Using this technique, Ward et al. (2005) have shown, during VAR of Inconel 718, that the arc centroid was most of the time off-centered and rotated around the electrode with a time constant between 20 and 40 seconds. Later, Woodside and King (2013) also reported an overall azimuthal motion of the arc for a Ti64 alloy but with a slightly smaller time period of 1-20 seconds and described three different arc distribution patterns. Such behaviour of the arc implies that the arc does not behave axisymmetrically at the macroscopic scale, which calls into question for the 2D axisymmetric modelling approach of the ingot growth generally used in the literature (Jardy and Ablitzer 2009).

The second approach used to investigate the arc at large time scales is based on optical techniques. Since the arc, which is essentially established at the electrode bottom surface, is not directly visible, it 
relies on observations, through the annular gap between the electrode and the crucible, of the light emitted by the arc and reflected by the free surface of the liquid pool. A straightforward method to monitor this light is to employ the supervision video cameras installed on top of VAR furnaces, yet it provides only qualitative results as the cameras are not calibrated. Using this technique, Jardy et al. (2013) have observed during zirconium alloy (Zy2) melts the existence of an asymmetrical arc distribution with a periodic ensemble arc motion. Recently, Aikin and Williamson (2009) introduced a new method using four calibrated photodiode sensors which were installed inside a laboratory scale VAR furnace. The signals of the four photodiodes were processed to estimate the location of the "average" arc position. Off-center distributions of the arc at a given instant during VAR of Inconel 718, in particular, were observed. The photodiode based optical technique proposed by Aikin and Williamson (2009) offers two main benefits. Similarly to the technique based on magnetic field measurement employed by Ward (2005) and Woodside and King (2013), it allows a quantitative analysis of the arc dynamics. Yet, the measurements are less complex to post-process and interpret than those obtained using the magnetic field based measurement technique.

The present paper seeks to investigate the dynamics of the arc during VAR of Ti64 alloy using a photodiode based optical technique similar to that proposed by Aikin and Williamson (2009), but implemented in a full scale VAR furnace. The location of the arc luminosity centroid is derived from the signals recorded by the photodiodes.

\section{Experiments}

\subsection{Experimental setup}

The measurements were carried out inside a standard full scale VAR furnace in Timet Savoie Framatome melt shop at Ugine, France. The reflection of the light emitted by the arc from the top surface of the molten pool was monitored using four photodiodes (Thorlabs PDA36A) that were arranged $90^{\circ}$ to one another and viewing down the annulus gap between the electrode and the crucible. The photodiodes were inserted into specifically developed modules that were mounted on a ring attached to the inner wall of the furnace (figure 2). The photodiode had a 3.6 by $3.6 \mathrm{~mm} \mathrm{Si}$ 
detector with a spectral response of 35 to $1100 \mathrm{~nm}$ and a switchable gain-amplifier. An interferential filter with a maximal transmission between $335 \mathrm{~nm}$ and $610 \mathrm{~nm}$ was placed in front of each photodiode to eliminate from the recorded signal any contribution due to thermal radiation of the molten pool. Each photodiode was also equipped with a quartz glass window and a $1 \mathrm{~mm}$ diameter pinhole to reduce the field of view of the photodiode. The pinhole dimensions were defined as such to prevent any overlapping of photodiode view fields from the beginning to the end of the melt. Finally, each photodiode was mounted on a bi-axis goniometer in order to finely tune its orientation. The photodiode signals and the power cables were transferred outside the furnace through a vacuum feed through. The photodiode outputs were recorded at $10 \mathrm{kHz}$ using a National Instrument NI9222 voltage input module connected on a National Instrument CDAQ-9174 board.

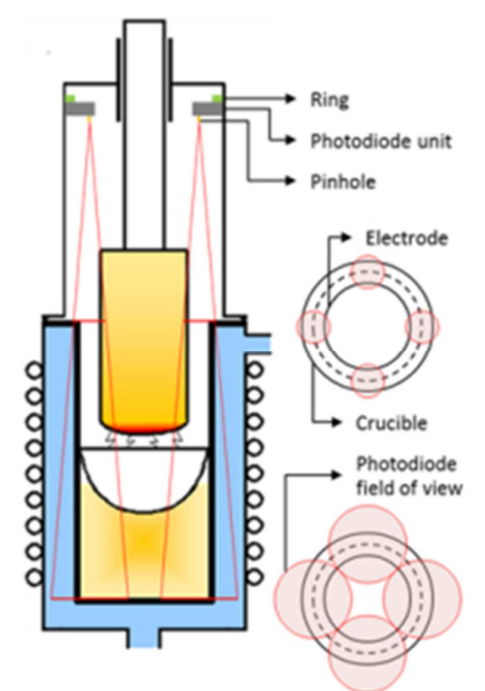

Figure 2: Schematic representation of the instrumentation of a VAR furnace with photodiodes showing the view field of each photodiode.

\subsection{Data processing}

First, a $0.5 \mathrm{~s}$ moving average was applied to the photodiode recorded data in order to remove high frequency fluctuations of the luminosity, followed by a resampling at $100 \mathrm{~Hz}$. Then the background noise was subtracted to the photodiode signal. Finally, in order to be able to compare the signals from the four photodiodes, calibration coefficients were applied to each signal. The calibration coefficients used in the present work are based on the assumption that the arc is on average evenly distributed throughout the melt plateau. This assumption is suggested by observations of the electrode tip after a 
melt interruption reported in the literature (e.g. Zanner et al. 2005), showing that the electrode tip remains relatively flat under standard conditions (namely diffuse arc conditions). Consequently, the average luminosities of the arc monitored by the four photodiodes during the melt plateau are considered to be identical. The position of the arc luminosity centroid was identified to the centroid of the four photodiode signals. The centroid position $\left(\mathrm{x}_{\mathrm{g}}, \mathrm{y}_{\mathrm{g}}\right)$ was calculated as:

$$
\begin{aligned}
& \mathrm{x}_{\mathrm{g}}=\sum\left(\mathrm{x}_{\mathrm{i}} \mathrm{V}_{\mathrm{i}}\right) / \sum \mathrm{V}_{\mathrm{i}} \\
& \mathrm{y}_{\mathrm{g}}=\sum\left(\mathrm{y}_{\mathrm{i}} \mathrm{V}_{\mathrm{i}}\right) / \Sigma \mathrm{V}_{\mathrm{i}}
\end{aligned}
$$

where $V_{i}$ is the voltage recorded by the photodiode $i$, whose horizontal coordinates are $\left(x_{i}, y_{i}\right)$.

\subsection{Melt parameters}

Measurements were performed during the melt of a $5400 \mathrm{~kg}$ Ti64 electrode with a $750 \mathrm{~mm}$ average diameter into a secondary ingot with an average diameter of $830 \mathrm{~mm}$. An alternated stirring sequence with a high magnetic field and a short period of 0.153 tau (time arbitrary unit) was applied. For confidentiality reasons, the melt parameters cannot be displayed. Therefore, arbitrary units are used.

\section{Results}

\subsection{Time evolution of the photodiode signals}

Figure 3a presents a typical time evolution of the voltage signals delivered by the four photodiodes recorded at same moments during the melt. In order to help interpretation of those signals and to evaluate the relevance of the data provided by the photodiodes for characterizing arc luminosity fluctuations, we present in figure $3 b$, for the same time interval as in figure $3 a$, data obtained by analysing video images of the melt recorded using two synchronized video cameras positioned on top of the furnace.

The main steps of the video image processing procedure were as follows. First, the recorded films were split into a series of images. From these images, a new series of images was then generated by applying a $2 \mathrm{~s}$ moving average in order to suppress high frequency fluctuations related to individual cathode spot behaviour. Then, a given region of interest was extracted from each image and all the results were put side by side to build a temporal sequence, enabling the quantification of the average luminosity in this 
region as a function of time. In figure $3 b$, we present the results of such processing for six regions of interest (three regions for each camera). The location of the four photodiodes with respect to the six regions of interest is given in figure 4.

(a)

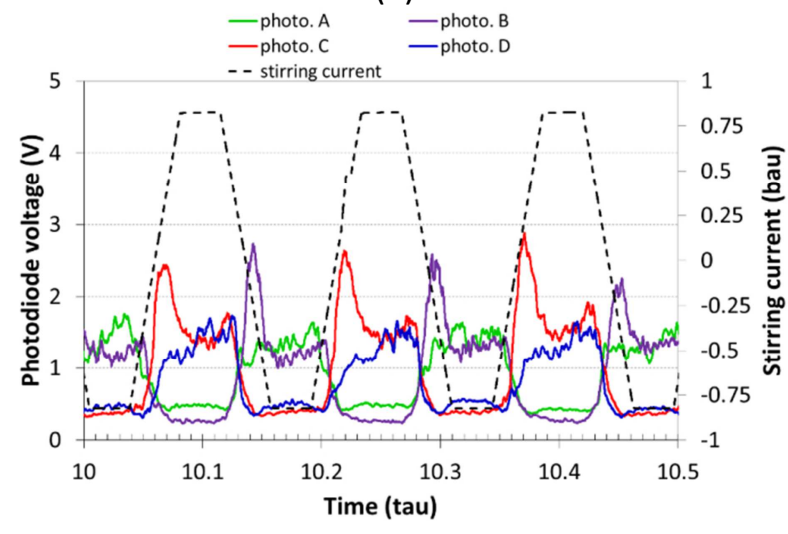

(b)

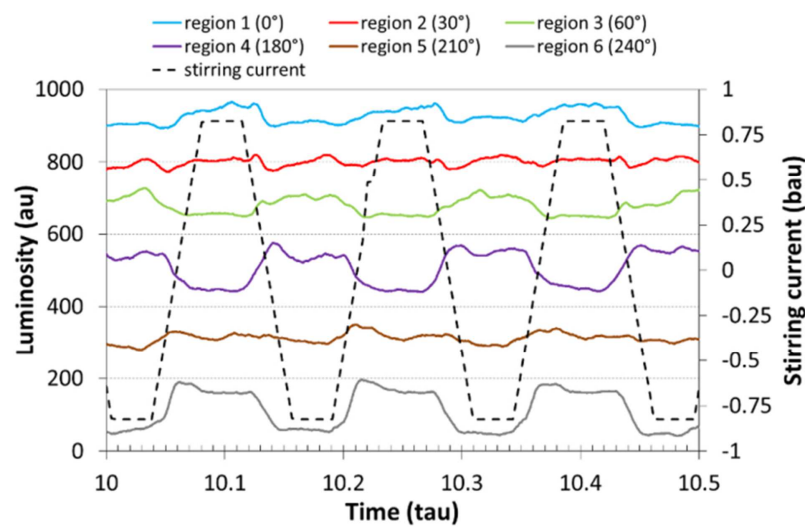

Figure 3: Typical time evolutions during the same time interval of (a) the four photodiode signals and (b) the arc luminosity analyzed from video recordings.

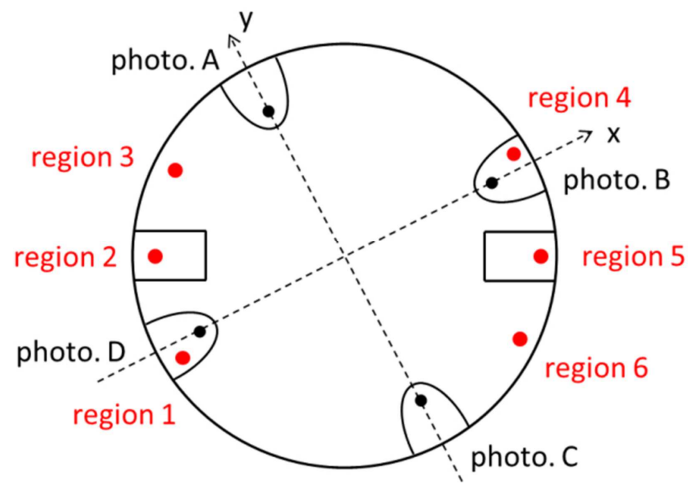

Figure 4: Location of the photodiodes with respect to the six regions of interest considered for the arc luminosity analysis based on video recordings.

For both sets of results (i.e. photodiode and video measurements), the signals vary strongly during the stirring ramps, whereas during the stirring plateaus the signals remain nearly constant, with a signal value being either high or low depending on the stirring polarity. High voltage values are observed for photodiodes $C$ and $D$ in the case of a positive polarity plateau, and for photodiodes $A$ and $B$ in the case of a negative polarity plateau. Results from video recording exhibit an evolution of the arc luminosity consistent with that of the voltage delivered by the photodiodes. A strong light emission is observed in regions 1 and 6 when the stirring polarity is positive, and in regions 3 and 4 when the stirring polarity is negative. However, in contrast to the behaviour of photodiodes B and C signals, regions 4 and 6 do not 
exhibit a luminosity peak before levelling off to a high luminosity value. The reasons for such a result are not well understood. This might be related to the very small size of the regions of interest selected on the video images compared to that of the regions analyzed using the photodiodes (as displayed in figure 2). Some localized fluctuations of the luminosity might thus be visible only on the photodiode signals. It might be also related to the fact that the sensitivity of the photodiodes to the luminosity fluctuations is much greater than that of the video cameras used in the present investigation.

As will be discussed later in more detail from the results of the calculation of the arc centroid position, the variations of the photodiode and video signals observed in Figure 3 reflect a different dynamic behaviour of the arc during stirring ramps and during stirring plateaus. The fact that the signals remain nearly constant during the stirring plateaus is an indication that the arc centroid remains relatively stable during this time interval. In contrast, the fact that the signals vary strongly during the stirring ramps (and in a different manner depending on the analyzed region) is a sign that the location of the arc centroid is moving during this time interval.

The four photodiode signals presented in figure $3 a$ exhibit clear periodic variations. A spectral analysis reveals that the primary and secondary periods correspond to the period and half-period values of the stirring sequence, indicating a strong correlation between the evolution of the arc luminosity recorded by the photodiodes and the stirring sequence.

\subsection{Patterns described by the arc luminosity centroid}

The main finding of the present study is that the motion of the arc luminosity centroid describes three regular patterns during the melt. Figure 5 illustrates the presence of these patterns (thereafter called A, B and C) during the whole melt. The hatched area corresponds to a moment when no centroid position can be calculated as at least one of the photodiode signals was not received by the computer. At some moments in the melt, no regular pattern can be deduced from the measured position of the centroid. During the melt start-up, the centroid position is highly variable. This behavior can be related to 
possible heterogeneities in the electrode tip surface, as the liquid film is not stable enough. Such heterogeneities could have a high influence on the creation and motion of the cathode spots at the microscale, resulting in the absence of a regular centroid position. The other two moments, when no regular pattern was observed, correspond to transitions between stable patterns.

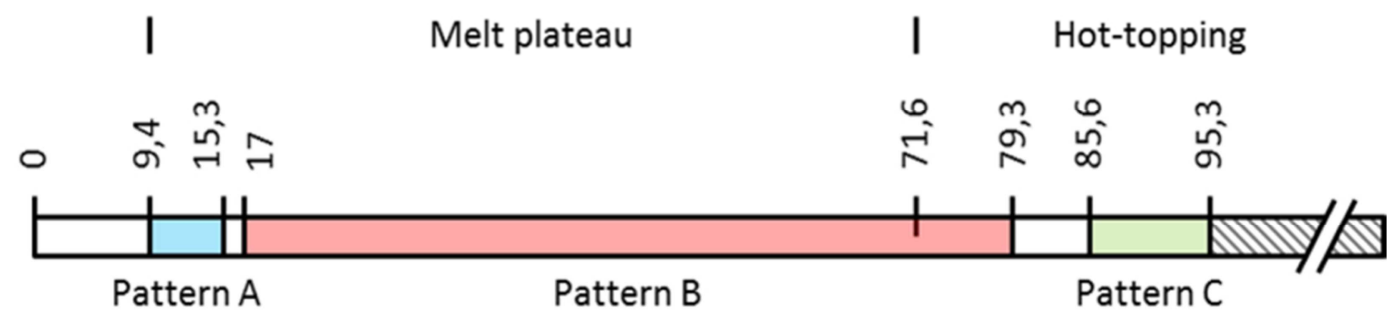

Figure 5: Timeline for the whole melt (in tau), with an indication of the arc patterns.

As mentioned in section 3.1, the results of the analysis of the photodiode signal clearly show a high correlation with the stirring sequence. Therefore, a colour code indicating the precise moment in the stirring sequence is used in the following figures, which detail the main characteristics of the different patterns. Figure 6 presents this colour code.

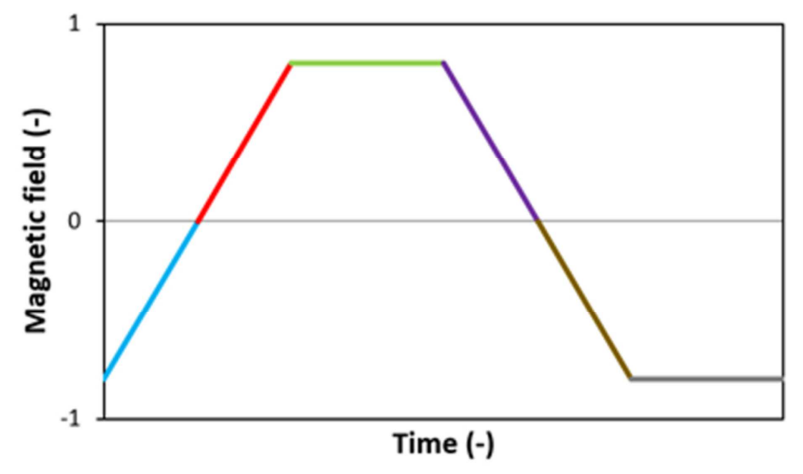

Figure 6: Colour code of the precise moment in the stirring sequence, used in figures 7-9.

\section{Pattern A}

The main characteristics of pattern A are illustrated in Figure 7, which presents time evolutions of the polar coordinates and velocity of the arc luminosity centroid, as well as a typical trajectory of the centroid during a single stirring period. 
(a)

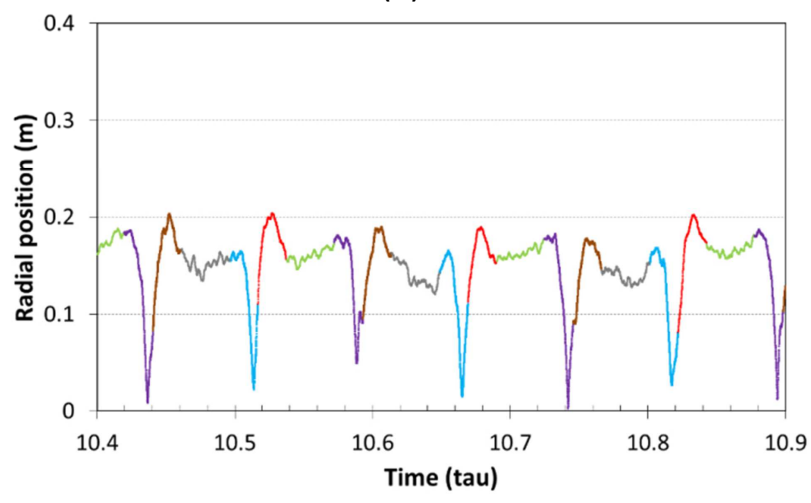

(c)

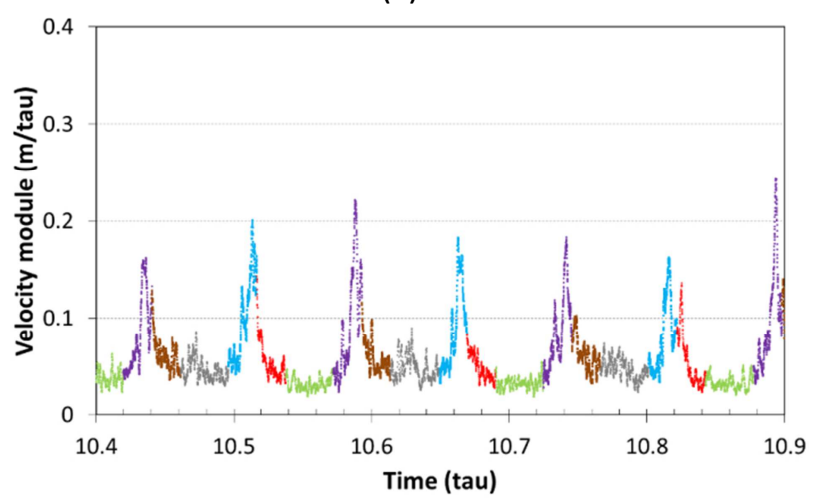

(b)

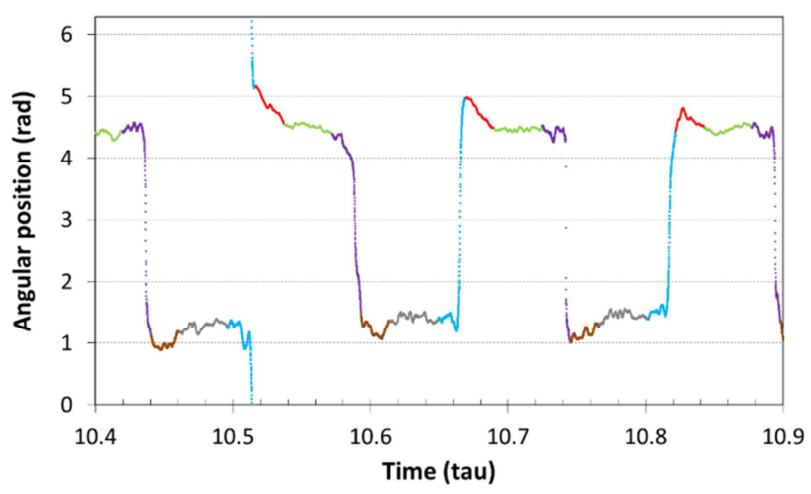

(d)

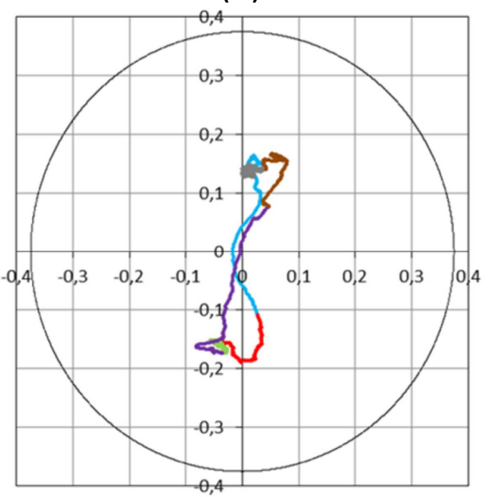

Figure 7: Pattern A: Typical temporal evolution of centroid (a) radial position, (b) angular position and (c) velocity. (d) Typical trajectory of the arc luminosity centroid during a single stirring period.

This pattern is characterized by a back and forth motion of the centroid between two off-centered regions of higher stability that are almost diametrically opposite. The centroid moves during the stirring ramps and keeps relatively stable in one of the areas during the stirring plateaus. Within each stirring ramp, the centroid first moves from one area toward the electrode centre then from the centre toward the second area. This motion is mostly linear but the centroid can move toward an off-center position before establishing in a stable area. Most of the motion takes place during the decrease of the magnetic field intensity.

The centroid velocity is generally the largest when the magnetic field intensity decreases and remains low through the stirring plateaus. When the magnetic field decreases, the velocity increases, reaching a maximum value before the magnetic field intensity cancels. When the magnetic field increases, the velocity lowers progressively and eventually levels off during the next stirring plateau. 


\section{Pattern B}

The main characteristics of pattern $B$ are illustrated in Figure 8 , which presents time evolutions of the polar coordinates and velocity of the arc luminosity centroid, as well as a typical trajectory of the centroid during a single stirring period.

(a)

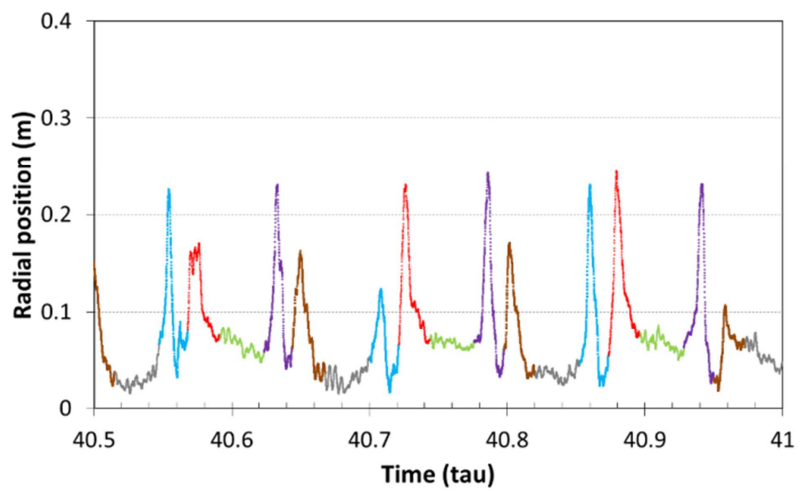

(c)

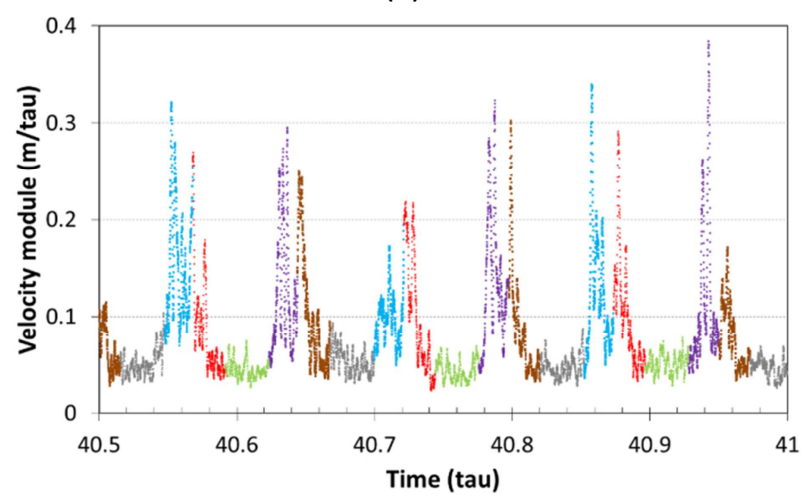

(b)

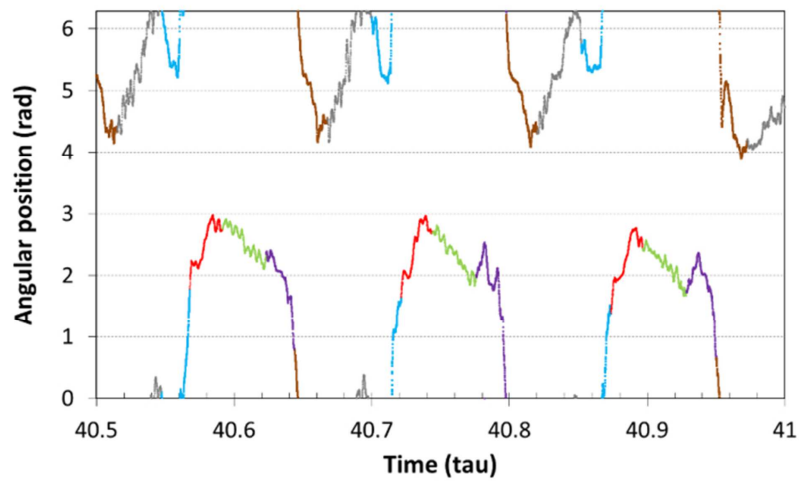

(d)

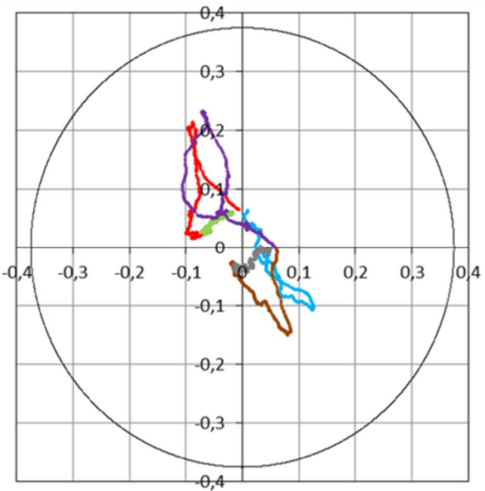

Figure 8: Pattern B: Typical temporal evolution of centroid (a) radial position, (b) angular position and (c) velocity. (d) Typical trajectory of the arc luminosity centroid during a single stirring period.

The pattern B is also characterized by a back and forth motion between two areas of higher stability. However, this motion is not linear, as in pattern A, but follows an elliptic path. Again, the centroid is almost immobile during the stirring plateaus and each area is associated with a given polarity of the coil current. However, we can often observe an angular drift during the stirring plateau and a slight radial motion toward the crucible at the end of the plateau. The motion between the two areas takes place during the stirring ramps, with more of its half when the magnetic field is decreasing (the highest velocity is observed at those moments, and it is higher than in pattern A). Its angular direction (clockwise vs. counter-clockwise) generally depends on the direction of inversion of the stirring polarity. 
Like in pattern $A$, the centroid velocity is low and stable during the stirring plateaus and reaches a maximum during the stirring ramps. The evolution of the velocity during the polarity reversal is different from that observed in pattern A. Two peaks are mostly observed. These peaks occur mostly when the magnetic field decreases. The minimum value of the velocity reached between the two peaks is correlated to the minimal radial position of the centroid.

\section{Pattern C}

Finally, figure 9 presents a time evolution of the centroid location and its velocity during pattern C. This pattern is significantly different from the two previous ones. The photodiode signals centroid is almost centered during the whole stirring sequence, with an average radial position of $0.03 \mathrm{~m}$. Its angular position shows a higher variation than in patterns $A$ and $B$, while the stirring plateaus are the moments of lower motion intensity. The centroid velocity is irregular. During the stirring plateaus, the velocity is greater than that observed in patterns $\mathrm{A}$ and $\mathrm{B}$. The maximum velocity reached during the stirring ramps is similar to that observed in pattern A and slightly smaller than that observed in pattern $\mathrm{B}$.

(a)

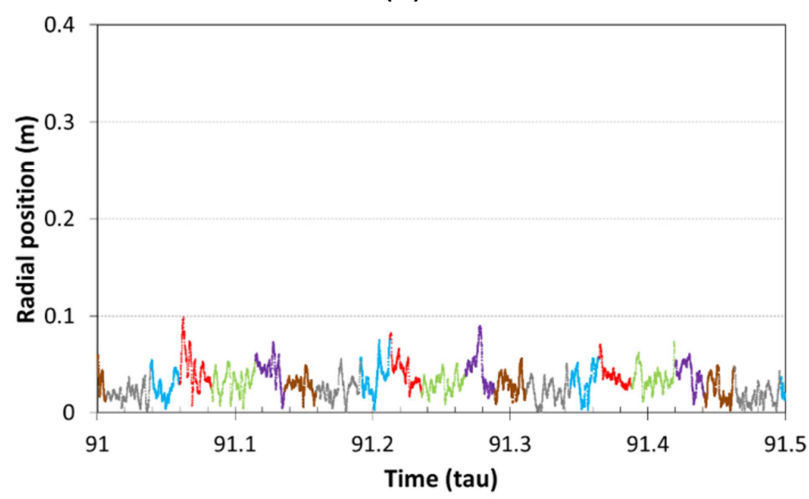

(b)

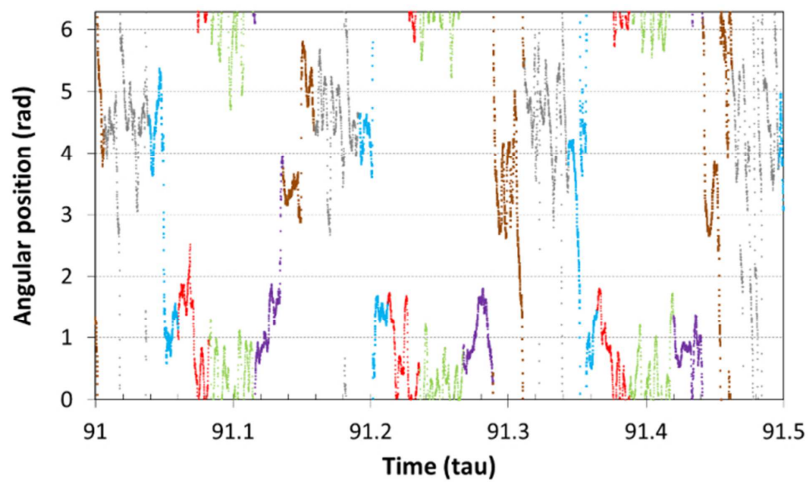

(c)

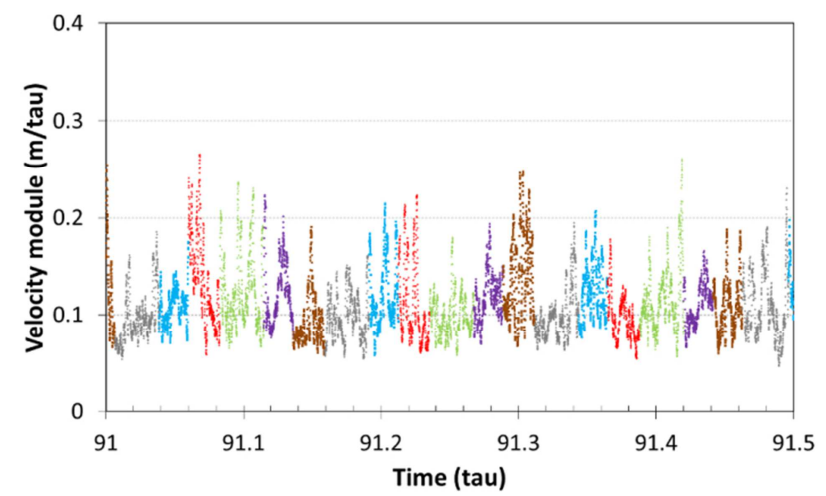

Figure 9: Pattern C: Typical temporal evolution of centroid (a) radial position, (b) angular position and (c) velocity. 
In order to summarize the results previously detailed, figure 10 represents a typical probability of the presence of the centroid on the electrode during 0.5 tau for the three patterns. The value of each pixel in figure 10 is proportional to the time duration the arc luminosity centroid was located on that pixel during the considered time interval. The main characteristics of the centroid patterns described above may be clearly identified on figure 10 .

(a)

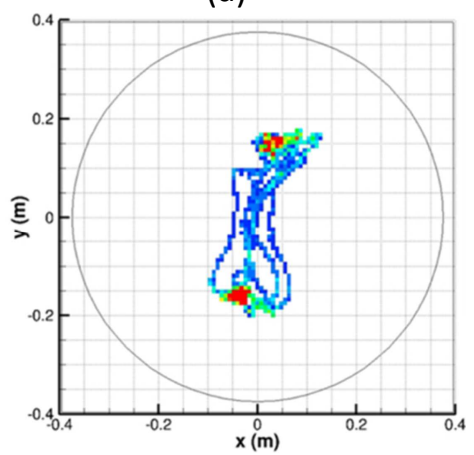

(b)

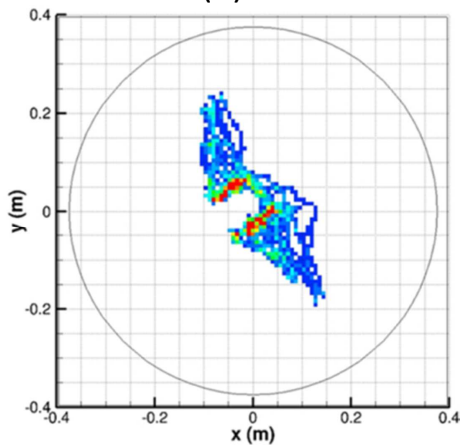

(c)

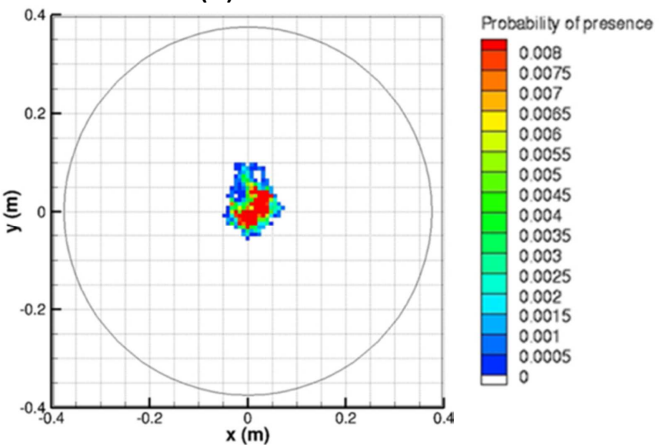

Figure 10: Probability of the presence of the centroid on the electrode during 0.5 tau for the patterns (a) A, (b) B and (c) C.

\subsection{Evolution of the location of the stable regions associated to the stirring plateaus}

As described above, for the three identified patterns, the arc luminosity centroid is located in a relatively stable region during the stirring plateaus. These stable regions evolve through the melt, as is shown in figure 11a, which presents the evolution through the whole melt of the average angular position of the centroid during the stirring plateaus, differentiating the plateaus according to the stirring polarity. The two stable regions remain fixed during pattern A, but their angular positions vary significantly during pattern B. This variation in the case of pattern B is quite periodic, with a characteristic primary period of 8.89 tau, which corresponds to 60 times the stirring period. The reason for the higher scattering of the angular position in the pattern $B$ as compared to patterns $A$ and $C$ remains unclear. It may be related to changing conditions in the contact region between the pool periphery and the crucible wall during the melt, affecting in particular local heat flux and electric current flow transferred radially towards the crucible wall. 
Similarly to figure $11 \mathrm{a}$, figure $11 \mathrm{~b}$ presents the evolution through the whole melt of the average radial position of the centroid during the stirring plateaus. Although the two stable regions are strongly offcentered at the beginning of the melt, they remain close to the electrode axis during most part of the melt. The stirring polarity apparently has no significant influence on their radial positions, which differ on average only $1.7 \mathrm{~cm}$ except at the beginning of the melt. Given this result and the fact that the angular distance between the two stable regions remains close to $\pi$, the present analysis suggests that the two stable regions are always about diametrically opposite.

(a)

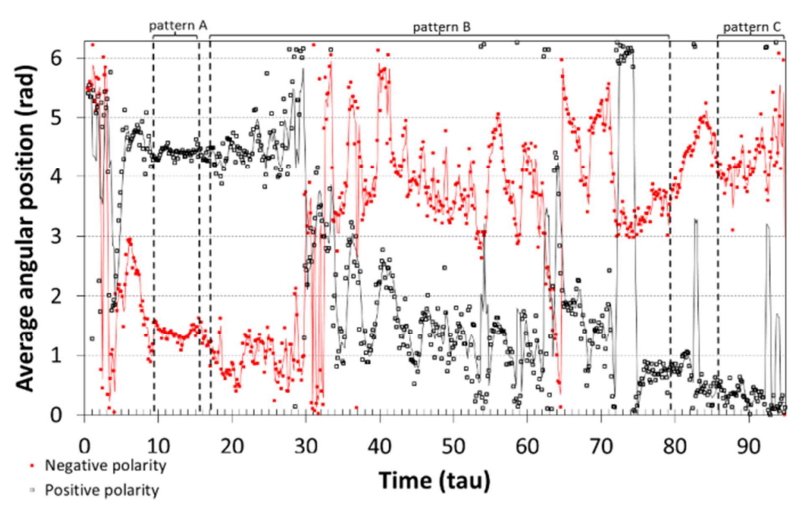

(b)

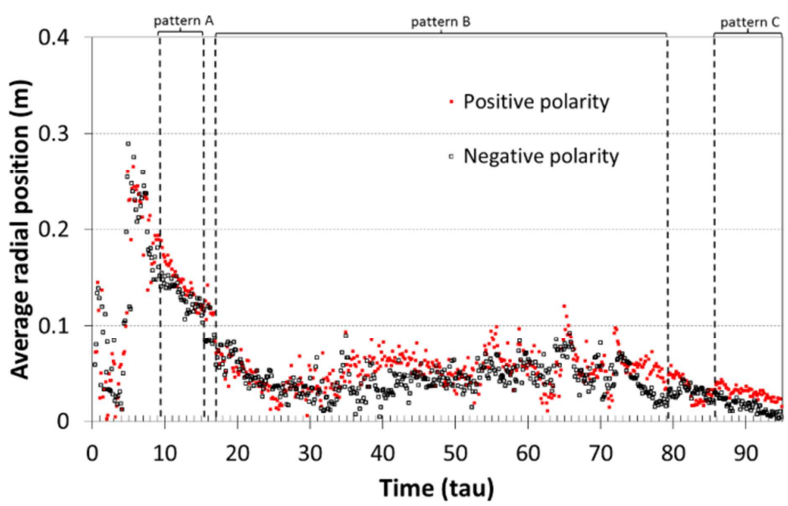

Figure 11: Average (a) angular and (b) radial positions of the arc luminosity centroid during stirring plateaus.

\section{Discussion}

Two phenomena could explain the observed behaviour of the centroid of the four photodiode signals. The first phenomenon is related to a local modification of the reflection angle at the liquid pool surface, whereas the second one is an ensemble motion of the arc. However, if variations in the arc light reflection on the free surface were the only reason of the apparent luminosity evolution, the offcentered locations of the stable areas in patterns A and B would not be compatible with a steady axisymmetric shape of the free surface during a stirring plateau. In addition, if a localized deformation of the surface was caused by an inversion in the coil current polarity, resulting in the luminosity patterns observed, the inertia of the liquid pool would probably cause some delay. Such a delay was not observed. The centroid starts moving immediately when the magnetic field starts to decrease. Therefore, we can conclude that the first possible explanation has a low probability and that the luminosity patterns observed during this melt were created by an ensemble motion of the electric arc. 
In the literature, the existence of an ensemble arc motion has already been reported for the case of the remelting of a nickel based alloy (Ward et al. 2005), a titanium alloy (Woodside et al. 2013), and a zirconium alloy (Jardy et al. 2013). We observe, however, a clear correlation between the arc motion and the stirring sequence, which was not described in previous papers.

The pattern $\mathrm{C}$ may be considered as a degenerate version of pattern $\mathrm{B}$ in the case of a low arc current. Indeed, a progressive transition between pattern $B$ and pattern $C$ is observed during the first stage of the hot-topping. This transition is characterized by a progressive reduction of the height of the luminosity peaks indicating a reduction of the off-centering of the arc. During the transition, two stable regions occupied, according to the stirring polarity, by the arc centroid during the stirring plateaus are still observed, with a non-linear motion of the centroid between the two regions during the stirring ramps. However, the inclination of the centroid to move towards off-centered positions during stirring ramps tends to disappear progressively. This interpretation of pattern $\mathrm{C}$ as a degenerate version of the pattern $B$ is also suggested by the strong similarity of the evolutions of the centroid angular position observed during patterns $B$ and $C$ (cf. figures $8 b$ and $9 b$ ). In both cases, the angular position varies mostly during the stirring ramps and remains relatively stable during the stirring plateaus.

Our results have shown the existence of complex arc dynamics, which are characterized on one hand by a stability of the arc during the stirring plateaus, with two diametrically opposite stable regions of the centroid linked to the polarity of the magnetic field, and on the other hand, by a motion of the centroid during the stirring ramps. Pattern A solely implies a linear translation motion between the two stable regions, whereas pattern $B$ involves a rotational motion. A complete understanding of the phenomena responsible for the observed arc dynamics is still lacking. Here we shall solely propose a tentative interpretation of the existence of the two stable regions, those two points appearing to be clearly linked to the imposed magnetic field. Other features of the observed arc dynamics (i.e. the translation or rotational motion between the two stable regions) shall be discussed in a future paper from 
additional observations made with the furnace video camera exploring a wider range of stirring conditions.

Two mechanisms may be considered to explain the existence and location of the two stable regions. The first mechanism is the Robson angular deviation of the cathode spots. According to works dedicated to vacuum arcs, under the presence of an axial magnetic field (such as the one imposed during a VAR melt), the motion of each cathode spot, which is preferentially directed towards the edge of the cathode, is subjected to an angular deflection called Robson drift (Robson 1959) thus resulting in a spiral type motion of each spot. The orientation of the deflection depends on the direction of the axial magnetic field. Therefore, the direction of the spot deflection is reversed when the stirring polarity is reversed. The existence of two diametrically opposite regions could be at least partially related to this phenomenon.

The second mechanism, that may be considered to interpret the existence of the two stable regions, is related to the homogeneity of the magnetic field generated by the stirring coil around the furnace. Analysis of the cathode spot distribution at the cathode surface in vacuum circuit breakers under the presence of an axial magnetic field with a non homogeneous radial distribution has shown that the spots tend to locate preferentially in the regions with a weak or strong intensity of the magnetic field depending on the conditions (Chaly et al. 2007). Such a behavior has been interpreted from the dependence of the arc voltage with the intensity of the magnetic field ( $U$ shaped curve) and by invoking the Steenbeck principle, according to which the spot distribution evolves to minimize the arc voltage.

Assuming (despite very different operating conditions) that the spot distribution in a VAR furnace is controlled by similar mechanisms, the off-centering of the arc during the stirring plateau could be the sign that the radial distribution of the magnetic field is non homogeneous. However, a delicate point to explain is the existence of two diametrically opposite regions correlated to the stirring polarity. The 
reversal of the stirring polarity alone does not justify a priori the displacement of the stable region to a diametrically opposite location.

Two main origins of the non homogeneity of the magnetic field may be identified. The helical coil of wire generating the magnetic field may have been irregularly or loosely wrapped around the crucible. Yet it is unlikely that such a defect may lead to a different intensity of the magnetic field according to the stirring polarity. Another origin of the non homogeneity of the magnetic field may be that the symmetry axis of the coil was improperly aligned with the symmetry axis of the electrode. This defect would not cause a modification of the intensity of the axial magnetic field when the polarity is reversed; however, it would be responsible for the existence of an additional magnetic field component, tangential to the electrode, whose direction would reverse according to the stirring polarity. As shown in figure 12 , the modification of the direction of the tangential magnetic field influences the direction of the Robson drift and could lead to a reversal of the direction of the cathode spot motion. This may play a role in the displacement of the centroid stable region to a diametrically opposite location.

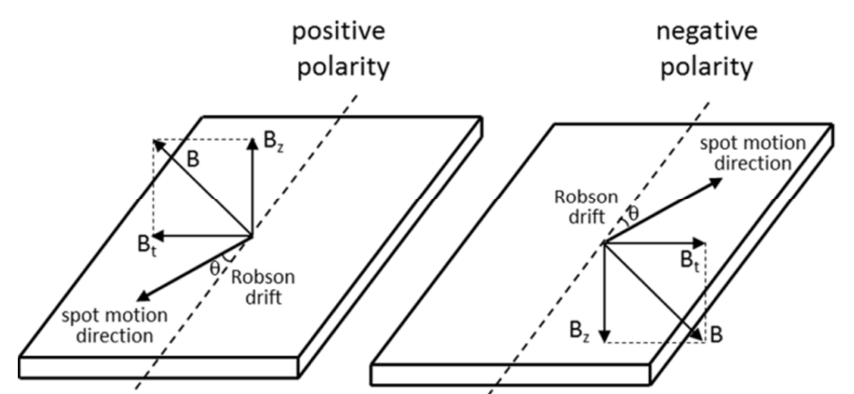

Figure 12: Schematic representation of spot motion direction with different stirring polarities in case of an imperfect alignment of the axes of the stirring coil and the electrode.

The arc dynamics reported in the present study may have several implications on the ingot growth and solidification, and consequently on the quality of the final product. The most important implication is expected to be the effect of the arc on the hydrodynamics of the liquid metal in the pool. As mentioned previously, the spatial and temporal distribution of the arc controls the inputs of electric current and energy at the ingot top, which determine, through buoyancy forces and electromagnetic forces, the motion of the metal in the molten pool. The existence of an ensemble arc motion as observed in the 
present study means that the distributions of electric current and energy at the ingot top are time varying and non-axisymmetric, which is most likely to cause transient and non-axisymmetric flow patterns in the pool. This will in particular certainly induce a non-axisymmetry in the shape of the molten pool. It is also possible that it will impact the overall mixing of the metal in the pool, and in this way influence the gradients of temperature and alloy elements concentration at the solidification front, which play an important role on the solidification microstructure and the formation of macrosegregation in the final ingot.

In addition, the transient and non-axisymmetric arc distribution induced by the existence of an ensemble arc motion may also affect the solidified metal region formed at the periphery of the ingot in contact with the crucible wall (referred hereafter as the shelf), and in this way influence the ingot sidewall quality. As mentioned above, the arc controls the thermal conditions at the ingot top. The proximity or the moving away of the arc may significantly change the local thermal conditions, causing the local remelting or growth of the shelf. Hence, as observed experimentally by Ward (2005), the arc motion may be responsible for important fluctuations of the thickness of the shelf, which could be relevant to the final structure of the ingot skin. Moreover, localized remelting of the shelf may cause the fall of fragment of metal in the pool, which if not remelted may be a source of defect in the final ingot.

Hence the existence of an asymmetrical arc motion is most likely to create the conditions to favor the production of an ingot with non-axisymmetric properties and the formation of defects. It follows from these considerations that, since the arc centroid remains close to the electrode axis in pattern C (which is indicative of a relatively axisymmetric arc distribution), one expects the pattern $C$ to be more desirable than patterns $A$ and $B$. 
Finally, it is important to mention that the existence of an asymmetrical arc motion has important implications for the modelling of the VAR ingot. It puts under question the classical assumption made in most models of a time-averaged axisymmetric and quasi-stationary arc distribution (Jardy and Ablitzer 2009). Models based on a 2D axisymmetric geometry might thus be not sufficient. A realistic representation of the arc behaviour would require turning to models based on a 3D geometry.

\section{Conclusions}

A quantitative study of the arc dynamics during an industrial VAR melt of a Ti64 alloy, with an imposed alternated electromagnetic stirring of high intensity, has been carried out based on the analysis of the arc luminosity using photodiodes. Photodiode data were corroborated with video camera recordings, demonstrating the ability of the photodiode instrumentation to be used as a diagnostic tool of the arc motion. The main interest of the photodiode instrumentation is the possibility to quantitatively study the arc dynamics throughout the whole melt.

Calculation of the position of the arc luminosity centroid has revealed that this centroid described three regular patterns during the investigated melt. Pattern $A$ appeared during a short time interval at the beginning of the melt plateau. After a short transition phase, pattern B was observed before disappearing shortly after the beginning of the hot-topping. In both cases, a motion of the centroid (linear in the case of pattern A and elliptic in the case of pattern B) was observed during the reversal of the stirring polarity, whereas the centroid remains quite stable during the stirring plateaus, with two diametrically opposite stable regions of the centroid linked to the polarity of the magnetic field. During the third pattern (C), which was observed during the hot-topping, the arc centroid was always located a very small distance from the electrode symmetry axis. A tentative interpretation of some of the mechanisms responsible for the existence of two stable regions has been proposed, considering the influence of the imposed magnetic field on the evolution of the cathode spot distribution. Yet, more research, involving in particular a detailed characterization of the possible non-homogeneity of the 
magnetic field generated by the stirring coil and complementary measurements for a wider range of operating conditions, would be needed to confirm those mechanisms and to achieve a thorough understanding of the various arc behaviours identified.

The transient and asymmetrical arc distribution at the ingot top caused by the existence of an ensemble arc motion has the potential to have important implications on the liquid metal flow and the solidification conditions, as well as on the local structure of the ingot skin. It may thus affect in the end the solidification microstructure and the formation of macrosegregation in the final ingot, as well as the ingot sidewall quality. Those points need to be studied into more details in the future.

\section{References}

Aikin, R.M., Williamson, R.L., 2009. A new optical emission monitor for vacuum arc remelting. In: International Symposium on Liquid Metal Processing and Casting, Santa Fe, NM, pp. 65-74.

Chaly, A.M., Logatchev, A.A., Zabello, K.K., Shkol'nik, S.M., 2007. High-current vacuum arc in a strong axial magnetic field. IEEE Trans. Plasma Sci. 35, 939-945.

Chapelle, P., Bellot, J.-P., Jardy, A., Czerwiec, T., Robbe, X., Champin, B., Ablitzer, D., 2000. An experimental study of the electric arc during vacuum arc remelting. High Temp. Mat. Proc. 4, 493-506.

Chapelle, P., Noël, C., Risacher, A., Jourdan, J., Jardy, A., Jourdan, J., 2014. Optical investigation of the behavior of the electric arc and the metal transfer during vacuum remelting of a Ti alloy. J. Mat. Process. Technol. 214, 2268-2275.

Choudhury, A., 1990. Vacuum metallurgy, A.S.M. Int..

Jardy, A., Ablitzer, D., 2009. Mathematical modelling of superalloy remelting operations. Materials Science and Technology 25, 163-169.

Jardy, A., Chapelle, P., Malik, A., Bellot, J.-P., Combeau, H., Dussoubs, B., 2013. Arc behaviour and cathode melting process during VAR: an experimental and numerical study. ISIJ Int. 53, 213220.

Robson, A.E., 1959. The motion of an arc in a magnetic field. In: Proc. of 4th International Conference on Ionization Phenomena in Gases, Uppsala, Sweden, pp. 346-349.

Shevchenko, D.M., Ward, R.M., 2008. Liquid metal pool behavior during the vacuum arc remelting of INCONEL 718. Metall. Mater. Trans. B 40, 263-270.

Ward, R.M., Daniel, B., Siddall, R.J., 2005. Ensemble arc motion and solidification during the vacuum arc remelting of a nickel based superalloy. In: International Symposium on Liquid Metal Processing and Casting, Santa Fe, NM, pp. 49-56.

Woodside, C.R., King, P.E., Nordlund, C., 2013. Arc distribution during the vacuum arc remelting of Ti6Al-4V. Metall. Mater. Trans. B 44, 154-165. 
Zanner, F.J., 1979. Observation of the vacuum arc and metal transfer during vacuum consumable arc remelting. In: Bhat, G.K., Schlatter, R. (Eds.), Proc. Int. Conf. on Special Melting. American Vacuum Society, pp. 417-427.

Zanner, F., Williamson, R., Erdmann, R., 2005. On the origin of defects in VAR ingots. In: International Symposium on Liquid Metal Processing and Casting, Santa Fe, NM, pp. 13-27. 


\section{Figure captions}

Figure 1: VAR process schematic representation.

Figure 2: Schematic representation of the instrumentation of a VAR furnace with photodiodes showing the view field of each photodiode.

Figure 3: Typical time evolutions during the same time interval of (a) the four photodiode signals and (b) the arc luminosity analyzed from video recordings.

Figure 4: Location of the photodiodes with respect to the six regions of interest considered for the arc luminosity analysis based on video recordings.

Figure 5: Timeline for the whole melt (in tau), with an indication of the arc patterns.

Figure 6: Colour code of the precise moment in the stirring sequence, used in figures 7-9.

Figure 7: Pattern A: Typical temporal evolution of centroid (a) radial position, (b) angular position and (c) velocity. (d) Typical trajectory of the arc luminosity centroid during a single stirring period.

Figure 8: Pattern B: Typical temporal evolution of centroid (a) radial position, (b) angular position and (c) velocity. (d) Typical trajectory of the arc luminosity centroid during a single stirring period.

Figure 9: Pattern C: Typical temporal evolution of centroid (a) radial position, (b) angular position and (c) velocity.

Figure 10: Probability of the presence of the centroid on the electrode during 0.5 tau for the patterns (a) A, (b) B and (c) C.

Figure 11: Average (a) angular and (b) radial positions of the arc luminosity centroid during stirring plateaus.

Figure 12: Schematic representation of spot motion direction with different stirring polarities in case of an imperfect alignment of the axes of the stirring coil and the electrode. 